\title{
ON THE UNIQUENESS OF CLASSICAL SEMICONJUGATIONS FOR SELF-MAPS OF THE DISK
}

\author{
PIETRO POGGI-CORRADINI
}

\begin{abstract}
We study the uniqueness properties of classical semiconjugations for analytic self-maps of the disk, by characterizing them as canonical solutions to certain functional equations. As a corollary, we obtain a complete description of all possible solutions to such equations.
\end{abstract}

\section{InTRODUCTION}

Given an analytic map $\phi$ defined on the unit disk $\mathbb{D}=\{z \in \mathbb{C}:|z|<1\}$ such that $\phi(\mathbb{D}) \subset \mathbb{D}$, and which is not an automorphism of $\mathbb{D}$, we will call it a self-map of $\mathbb{D}$. Often it will be convenient to change variables from $\mathbb{D}$ to the upper half-plane $\mathbb{H}=\{z \in \mathbb{C}: \operatorname{Im} z>0\}$ while keeping the same symbol $\phi$. We write $\phi_{n}$ for the $n$-th iterate of $\phi$. The pair $(\phi, \mathbb{D})$ could be thought to represent the semi-group (under composition) $\left\{\phi_{n}\right\}_{n=0}^{\infty}$, where $\phi_{0}=\operatorname{Id}_{\mathbb{D}}$.

When $\tau$ is an automorphism of $\mathbb{D}$, write $\tau \in \operatorname{Aut}(\mathbb{D})$, the dynamics $\left\{\tau_{n}\right\}$ can be understood completely by conjugating $\tau$ to rotations, translations, or dilations. This dynamics can be used as a model for arbitrary self-maps $\phi$ by finding semiconjugations. In certain cases, the automorphisms of $\mathbb{D}$ are not the right models and one must instead consider automorphisms of $\mathbb{C}$ (the type problem for simply connected Riemann surfaces is at the root of this fact, see [Cow81]). The present paper is mainly concerned with uniqueness and canonicity of the classical semiconjugations.

1.1. The classification. Recall that the automorphisms of $\mathbb{D}$ are divided into three categories:

(1) elliptic automorphisms, have one fixed point in $\mathbb{D}$ and can be conjugated to rotations $z \mapsto e^{i \theta} z$ in $\mathbb{D}$.

(2) hyperbolic automorphisms, have no fixed points in $\mathbb{D}$, two fixed points on $\partial \mathbb{D}$, and can be conjugated to dilations $z \mapsto T z, T>1$, on $\mathbb{H}$ (sometimes it's more convenient to conjugate to the dilations $z \mapsto t z, 0<t<1$ ).

(3) parabolic automorphisms, have no fixed points in $\mathbb{D}$, one fixed point in $\partial \mathbb{D}$, and can be conjugated to translations $z \mapsto z+1$ or $z \mapsto z-1$, on $\mathbb{H}$.

Date: November 23, 2005.

The author thanks Ch. Pommerenke for posing the question and for many stimulating discussions. 
In what follows we will include the identity map into the elliptic category.

Likewise if $\phi$ is a self-map of $\mathbb{D}$, we again have three categories (this follows from the classical Denjoy-Wolff Theorem and Julia's Lemma, see [BPC03] and references therein):

(1) elliptic type: $\phi$ has one fixed point $\zeta \in \mathbb{D}$ and can be conjugated to a map that fixes the origin.

(2) hyperbolic type: $\phi$ has no fixed points in $\mathbb{D}$, and has a fixed point $\zeta$ on $\partial \mathbb{D}$ (i.e. n. t. $-\lim _{z \rightarrow \zeta} \phi(z)=\zeta$ ) such that n.t. $-\lim _{z \rightarrow \zeta} \phi^{\prime}(z)=c$ exists and $0<c<1$. Such $\phi$ can be conjugated to $z \mapsto A z+p(z)$ on $\mathbb{H}$, with $A=1 / c>1, \operatorname{Im} p(z)>0$ and n. t. $\lim _{z \rightarrow \infty} p(z) / z=0$ (so that $\zeta$ corresponds to $\infty$ ).

(3) parabolic type: $\phi$ has no fixed points in $\mathbb{D}$, and has one fixed point $\zeta$ on $\partial \mathbb{D}$, such that n. t. $-\lim _{z \rightarrow \zeta} \phi^{\prime}(z)=1$. Such $\phi$ can be conjugated to $z \mapsto z+p(z)$ on $\mathbb{H}$, with $\operatorname{Im} p(z)>0$ and n.t. $-\lim _{z \rightarrow \infty} p(z) / z=0$.

In all three cases the point $\zeta$ is referred to as the Denjoy-Wolff point of $\phi$, and $\phi_{n}$ converges locally uniformly to $\zeta$. In particular, given any point $z$ the forward orbit $\left\{\phi_{n}(z)\right\}_{n=1}^{\infty}$ tends to $\zeta$.

The elliptic and parabolic types branch out further into subcases. In the elliptic case, if $\phi(0)=0$ and $\phi^{\prime}(0)=0$, then $\phi$ is superattracting, while if $\phi^{\prime}(0) \neq 0$, then $\phi$ is attracting and in this case $\left|\phi^{\prime}(0)\right|<1$ (sometimes this case is also called loxodromic). In the parabolic case, fix a point $z \in \mathbb{H}$ and consider the forward orbit $z_{n}=\phi_{n}(z)$. Then the hyperbolic step $s_{n}:=\rho_{\mathbb{H}}\left(z_{n}, z_{n+1}\right)$ decreases by Schwarz's Lemma, hence tends to a limit $s_{\infty}(z) \geq 0$. It is easy to see that if $s_{\infty}(z)=0$ for one point $z$, then it is zero for all $z \in \mathbb{H}$. Thus a parabolic map $\phi$ is called zero-step if $s_{\infty} \equiv 0$, and it is called non-zero-step if $s_{\infty}>0$ on $\mathbb{H}$.

1.2. The classical semiconjugations. In this paper we only consider the hyperbolic and parabolic cases. However, it should be possible to set up the same general framework for the elliptic case and for the semiconjugations that arise in the theory of backward orbits, see [PC00] and [PC03].

One of the main methods to produce semiconjugations is to renormalize the iterates. Here we recall Theorem 1 of [Pom79], see also [BPC03].

Fix a self-map of $\mathbb{H}$ of hyperbolic or parabolic type, which can therefore be written as

$$
\phi(z)=A z+p(z)
$$

$A \geq 1, \operatorname{Im} p(z)>0$ and n.t. $-\lim _{z \rightarrow \infty} p(z) / z=0$ (see the classification above in Section 1.1). Write $\operatorname{Aut}_{\infty}(\mathbb{H})=\{z \mapsto c z+b ; c, b \in \mathbb{R}, c>0\}$ for the automorphisms of $\mathbb{H}$ which fix $\infty$. Also write $z_{n}=\phi_{n}\left(z_{0}\right)=x_{n}+i y_{n}$. Then the automorphism $\gamma_{n}(z)=\left(z-x_{n}\right) / y_{n} \in \operatorname{Aut}_{\infty}(\mathbb{H})$ sends $z_{n}$ back to $i$. It is natural to consider the normalized iterates

$$
g_{n}=\gamma_{n} \circ \phi_{n} .
$$


Theorem A (Theorem 1 of [Pom79]). The limit $g=\lim _{n \rightarrow \infty} g_{n}$ exists locally uniformly in $\mathbb{H}$ and is a self-map of $\mathbb{H}$ with $g\left(z_{0}\right)=i$; the sequence $\gamma_{n} \circ \gamma_{n+1}^{-1}$ tends to $\alpha \in$ Aut $_{\infty}(\mathbb{H})$; and

$$
g \circ \phi=\alpha \circ g
$$

Moreover, if $A>1$ in (1.1) (i.e., $\phi$ is hyperbolic), then $\alpha(z)=A z+b$ (i.e. $\alpha$ is also hyperbolic), with $b=\lim _{n \rightarrow \infty}\left(x_{n+1}-x_{n}\right) / y_{n} \in \mathbb{R}$; if $A=1$ and $\rho_{\mathbb{H}}\left(z_{n}, z_{n+1}\right) \downarrow s_{\infty}>0$ (i.e., $\phi$ is parabolic non-zero-step), then $\alpha(z)=z+b$ (i.e. $\alpha$ is parabolic), with $b=\lim _{n \rightarrow \infty}\left(x_{n+1}-\right.$ $\left.x_{n}\right) / y_{n} \neq 0$; however, if $\phi$ is parabolic zero-step, then $\alpha(z)=z$ and $g \equiv 1$.

Remark 1.1. Notice that when $\phi$ is hyperbolic it can also be considered non-zero-step. In fact, it is proved in [Val54], see also Lemma 4 of [BPC03], that for every forward orbit of a hyperbolic self-map there is a Stolz angle at infinity, i.e. a sector $\{|\operatorname{Arg} z-\pi / 2|<\theta\}$ with $0<\theta<\pi / 2$, containing it. Then the non-zero-step property follows from (1.1).

Also in the hyperbolic case, every $b \in \mathbb{R}$ can arise as the constant coefficient for $\alpha$, by changing the starting point $z_{0}$ in the construction of $g$, see (2.9) in [BPC03].

Remark 1.2. If $\phi$ is parabolic non-zero-step, and $z_{n}=\phi_{n}\left(z_{0}\right)=x_{n}+i y_{n}$, the number $b \neq 0$ in Theorem $\mathrm{A}$ is equal to $\lim _{n \rightarrow \infty} \frac{x_{n+1}-x_{n}}{y_{n}}$. In particular, either $\lim _{n \rightarrow \infty} x_{n}=+\infty$ or $\lim _{n \rightarrow \infty} x_{n}=-\infty$. Also $y_{n}$ is strictly increasing, and $x_{n} / y_{n} \rightarrow 0$, i.e., either $\operatorname{Arg} z_{n} \rightarrow 0$ or $\operatorname{Arg} z_{n} \rightarrow \pi$ (see Remark 1 of [Pom79]). In the following we will always assume that $\lim _{n \rightarrow \infty} x_{n}=+\infty$.

1.3. Uniqueness questions. The natural questions that arise from Theorem A are the following.

Question 1.3. What happens if a different orbit, other than $z_{n}=\phi_{n}\left(z_{0}\right)$, is chosen when doing the renormalization (1.2)?

Question 1.4. To what extent are the functions $g$ and $\alpha$ obtained in Theorem A unique?

This last question was first raised by F. Bracci. His observation (see also [BPC03] p. 48) was that aside from Pommerenke's result, Valiron [Val31] had also obtain some semiconjugations using a different renormalization, C. Cowen in [Cow81] produced them using the Uniformization Theorem (hence with a more abstract approach), and recently Bourdon and Shapiro [BS97] also produced such maps under some regularity conditions on the self-map. Thus the question is to what extent are these maps 'essentially' the same map.

The goal of this paper is to clarify these questions and show how the classical semiconjugations are the canonical solutions for certain functional equations. 
1.4. Functional equations. We are interested in the following two functional equations: Forward Equation: Given the analytic self-map $\phi$ of $\mathbb{D}$, find an analytic self-map $\sigma$ of $\mathbb{D}$ and $\tau \in \operatorname{Aut}(\mathbb{D})$ such that

$$
\sigma \circ \phi=\tau \circ \sigma
$$

Backward Equation: Given the analytic self-map $\phi$ of $\mathbb{D}$, find an analytic self-map $\psi$ of $\mathbb{D}$ and $\eta \in \operatorname{Aut}(\mathbb{D})$ such that

$$
\psi \circ \eta=\phi \circ \psi
$$

The maps $\sigma$ and $\psi$ are called semiconjugations and the automorphisms $\tau$ and $\eta$ are called model automorphisms.

It turns out, however, that an important case, the so-called "parabolic zero-step" case naturally produces the following modified problem:

Planar Equation: Given the analytic self-map $\phi$ of $\mathbb{D}$, find an analytic map $\sigma$ with $\sigma(\mathbb{D}) \subset$ $\mathbb{C}$ and $\tau \in \operatorname{Aut}(\mathbb{C})$ such that

$$
\sigma \circ \phi=\tau \circ \sigma
$$

Definition 1.5. We write $\mathcal{F}(\phi)$ for the family of all non-trivial solution pairs $(\sigma, \tau)$ to $(1.3)$, i.e., we ask that $\sigma$ be non-constant.

For instance, the pair $(g, \alpha)$ in Theorem $\mathrm{A}$ is in $\mathcal{F}(\phi)$ when $\phi$ is hyperbolic or parabolic non-zero-step. Often, it will be enough to solve these equations in a conjugation class. In fact, if $\alpha, \beta \in \operatorname{Aut}(\mathbb{D})$, we have

$$
(\sigma, \tau) \in \mathcal{F}(\phi) \Leftrightarrow(\sigma \circ \alpha, \tau) \in \mathcal{F}\left(\alpha^{-1} \circ \phi \circ \alpha\right)
$$

and

$$
(\sigma, \tau) \in \mathcal{F}(\phi) \Leftrightarrow\left(\beta^{-1} \circ \sigma, \beta^{-1} \circ \tau \circ \beta\right) \in \mathcal{F}(\phi)
$$

In view of $(1.7)$, instead of $\mathcal{F}(\phi)$, it is enough to consider the families

$$
\mathcal{F}_{e}(\phi, \theta) \cup \mathcal{F}_{h}(\phi, T) \cup \mathcal{F}_{p}(\phi, \pm)
$$

where

- $\mathcal{F}_{e}(\phi, \theta)=\left\{\sigma: \mathbb{D} \rightarrow \mathbb{D} ; \theta \in[0,2 \pi) \mid \sigma \circ \phi=e^{i \theta} \sigma\right\}$.

- $\mathcal{F}_{h}(\phi, T)=\{\sigma: \mathbb{H} \rightarrow \mathbb{H} ; T>1 \mid \sigma \circ \phi=T \sigma\}$.

- $\mathcal{F}_{p}(\phi, \pm)=\{\sigma: \mathbb{H} \rightarrow \mathbb{H} \mid \sigma \circ \phi=\sigma \pm 1\}$.

Remark 1.6. With the notations of Theorem A, when $\phi$ is hyperbolic and $\alpha(z)=A z+b$ $(A>1)$, we can let $\beta(z)=z-b /(A-1)$ in $(1.7)$, then $\beta^{-1} \circ \alpha \circ \beta(z)=A z$, hence $\beta^{-1} \circ g \in \mathcal{F}(\phi, A)$. On the other hand, when $\phi$ is parabolic non-zero-step and $\alpha(z)=z+b$, we can let $\beta(z)=|b| z$ in $(1.7)$, then $\beta^{-1} \circ \alpha \circ \beta(z)=z+b /|b|$, hence $\beta^{-1} \circ g \in \mathcal{F}(\phi, \pm 1)$. 
Before solving these functional equations we must describe some further properties of the classical semiconjugations.

\section{Properties of the Classical semiconjugations}

2.1. Hyperbolic maximality. The following observation is due to Ch. Pommerenke (oral communication). The semiconjugations obtained in Theorem A are "maximal" with respect to the hyperbolic metric.

Proposition 2.1. Suppose $\phi$ is either hyperbolic or parabolic, as in (1.1), and let $g$ be the semiconjugation obtained in Theorem A. If $\phi$ is hyperbolic or parabolic non-zero-step, then for every $\sigma$ such that $(\sigma, \tau) \in \mathcal{F}(\phi)$ for some $\tau$, we have

$$
\rho_{\mathbb{H}}(\sigma(z), \sigma(w)) \leq \rho_{\mathbb{H}}(g(z), g(w)) \quad \forall z, w \in \mathbb{H} .
$$

If $\phi$ is parabolic zero-step, then $\mathcal{F}(\phi)$ is empty.

Proof of Proposition 2.1. Write $\tau_{n}$ for the $n$-th iterate of $\tau$. Then, given $z, w \in \mathbb{H}$,

$$
\begin{aligned}
\rho_{\mathbb{H}}(\sigma(z), \sigma(w)) & =\rho_{\mathbb{H}}\left(\tau_{n} \circ \sigma(z), \tau_{n} \circ \sigma(w)\right) \\
& =\rho_{\mathbb{H}}\left(\sigma \circ \phi_{n}(z), \sigma \circ \phi_{n}(w)\right) \\
& \leq \rho_{\mathbb{H}}\left(\phi_{n}(z), \phi_{n}(w)\right) \\
& =\rho_{\mathbb{H}}\left(g_{n}(z), g_{n}(w)\right),
\end{aligned}
$$

where $g_{n}$ is defined in (1.2). Proposition 2.1 now follows from Theorem A.

2.2. Univalence and covering properties of the classical semiconjugations. The semiconjugations obtained in Theorem A have the following useful univalence and covering properties. Suppose $\phi$ is as in (1.1), and suppose $\phi$ is either hyperbolic or parabolic nonzero-step. Let $(g, \alpha)$ be the pair of functions obtained in Theorem A, and let $\gamma_{n}$ be as in $(1.2)$.

Lemma 2.2 (Lemma 2 and Theorem 3 of [Pom79]). The sequence $\alpha_{n}^{-1} \circ g \circ \gamma_{n}^{-1}$ converges uniformly on compact subsets of $\mathbb{H}$ to the identity map on $\mathbb{H}$. Moreover, sequences $\rho_{k}, M_{k} \uparrow$ $+\infty$ can be chosen so that $g$ is univalent on the set

$$
U=\bigcup_{k=1}^{\infty} \bigcup_{n \geq M_{k}}\left(x_{n}+y_{n} \Delta\left(i, \rho_{k}\right)\right)
$$

Furthermore,

(a) If $\phi$ is hyperbolic, $U$ and $g(U)$ have an inner-tangent at infinity, i.e., given $\delta_{n} \downarrow 0$, there is $R_{n} \uparrow \infty$ such that $\left\{|z|>R_{n}, \delta_{n}<\operatorname{Arg} z<\pi-\delta_{n}\right\} \subset \Omega$. Finally, g has the 
following isogonality properties:

$$
\text { n. t. }-\lim _{z \rightarrow \infty} g(z)=\infty \text { and n. t. }-\lim _{z \rightarrow \infty} \frac{\operatorname{Arg} g(z)}{z}=0
$$

(b) If $\phi$ is parabolic non-zero-step and $x_{n} \rightarrow+\infty$, then the region $V=g(U)$, has a lateral-tangent at $+\infty$ with respect to $\mathbb{H}$, i.e., given $\delta_{n} \downarrow 0$ there is $t_{n} \uparrow+\infty$ such that $\left\{\operatorname{Re} z>t_{n}, \delta_{n}<\operatorname{Im} z<1 / \delta_{n}\right\} \subset V$.

Remark 2.3. When $\phi$ is parabolic non-zero-step and $z_{n}=x_{n}+i y_{n}$ is an orbit of $\phi$, then $y_{n}$ increases, so $y_{n} \uparrow L_{\infty} \leq+\infty$. In particular, if $L_{\infty}<\infty$, then $U$ itself has a lateral tangent at $+\infty$. But, if $L_{\infty}=\infty$, it is not clear. By Remark 2 of [Pom79], it turns out that $L_{\infty}<\infty$ if and only if $g$ has a finite angular derivative at infinity, i.e.,

$$
\text { n.t. } \lim _{z \rightarrow \infty} \frac{g(z)}{z}=\frac{1}{L_{\infty}} \text {. }
$$

Proof. Proof of Lemma 2.2 In [Pom79] it is proved that $\alpha_{n}^{-1} \circ g \circ \gamma_{n}^{-1} \rightarrow \operatorname{Id}_{\mathbb{H}}$ and that for any $\rho>0$ there is $m_{\rho} \in \mathbb{N}$ so that $g$ is univalent on

$$
\Omega\left(\rho, m_{\rho}\right)=\bigcup_{n \geq m_{\rho}}\left(x_{n}+y_{n} \Delta(i, \rho)\right)=\bigcup_{n \geq m_{\rho}} \gamma_{n}^{-1}(\Delta(i, \rho))
$$

Now, suppose a sequence $\rho_{k} \uparrow+\infty$ is given. Then $g$ is univalent on $\Omega\left(\rho_{1}, M_{1}\right)$. Suppose that $M_{1}<\cdots<M_{k}$ have been chosen so that $g$ is univalent on

$$
A_{k}:=\bigcup_{j=1}^{k} \Omega\left(\rho_{j}, M_{j}\right)
$$

Let $m_{\rho_{k+1}}$ be as in (2.4). Choose $M_{k+1}>m_{\rho_{k+1}}$ so large that, for all $n \geq M_{k+1}$,

$$
g\left(\gamma_{n}^{-1}\left(\Delta\left(i, \rho_{k+1}\right)\right)\right) \cap g\left(A_{k} \backslash \Omega\left(\rho_{k+1}, m_{\rho_{k+1}}\right)\right)=\emptyset .
$$

This can be done because $g \circ \gamma_{n}^{-1}-n b \rightarrow \operatorname{Id}_{\mathbb{H}}$. We claim that $g$ is univalent on $A_{k+1}$. In fact, suppose $g(z)=g(w)$ for $z, w \in A_{k+1}$. If $z \neq w$, then necessarily, $z \in A_{k+1} \backslash A_{k}$ and $w \in A_{k} \backslash \Omega\left(\rho_{k+1}, m_{\rho_{k+1}}\right)$ (or viceversa). Hence, $z \in \gamma_{n}^{-1}\left(\Delta\left(i, \rho_{k+1}\right)\right)$ for some $n \geq M_{k+1}$, and by (2.5), $g(z) \neq g(w)$, therefore $z=w$.

Furthermore, part (a) is well-known, see for instance p. 47 of [BPC03] and Lemma 5.1 of [PC00].

For part (b), let $(g, \alpha)$ be given by Theorem A, and assume $\lim _{n \rightarrow \infty} x_{n}=+\infty$, so that $\alpha(z)=z+b$ with $b>0$. Consider the half-strips $\mathbb{S}_{t}=\{\operatorname{Re} z>t, \delta<\operatorname{Im} z<1 / \delta\}$, and pick a large hyperbolic disk $\Delta(i, \rho)=\left\{z \in \mathbb{H}: \rho_{\mathbb{H}}(z, i)<\rho\right\}$, so that $\cup_{k \geq 0}(\Delta(i, \rho)+k b) \supset \mathbb{S}_{0}$. Now consider the compact set $B=\left\{z \in \mathbb{H}: \rho_{\mathbb{H}}(z, i) \leq 2 \rho\right\}$ and pick $\epsilon<\rho$. Then, by Lemma 2.2 there exists $N \in \mathbb{N}$ such that

$$
\rho_{\mathbb{H}}\left(\alpha_{n}^{-1} \circ g \circ \gamma_{n}^{-1}(z), z\right)=\rho_{\mathbb{H}}\left(g \circ \gamma_{n}^{-1}(z), \alpha_{n}(z)\right)<\epsilon
$$


for $n>N$, and uniformly for $z \in \partial B$. Hence, for $n>\max \left\{N, m_{\rho}\right\}$, and for every $w \in \Delta(i, \rho)$, we have that $g \circ \gamma_{n}^{-1}(\partial B)$ winds around $\alpha_{n}(w)$ exactly once, i.e., $\alpha_{n}(w) \in g(U)$. Thus $\mathbb{S}_{t} \subset g(U)$ for $t$ sufficiently large.

2.3. Orbits and canonical solutions. Given a point $z \in \mathbb{D}$, define $\left\{\phi_{n}(z)\right\}_{n=0}^{+\infty}$ to be the forward orbit starting at $z ; \cup_{n=0}^{+\infty} \phi_{n}^{-1}(z)$ the backward orbit starting at $z$. A sequence $\left\{w_{n}\right\}_{n=1}^{\infty}$ is called a backward iteration sequence if $\phi^{-1}\left(w_{n}\right) \neq \emptyset$ for $n=1,2,3, \ldots$, and $w_{n+1} \in \phi^{-1}\left(w_{n}\right)$.

Moreover, we say that $\cup_{k=0}^{+\infty} \cup_{n=0}^{+\infty} \phi_{n}^{-1}\left(\phi_{k}(z)\right)$ is the grand orbit generated by $z$. If $z, w$ belong to a grand orbit, then there are $n, k \in \mathbb{N}$ so that $\phi_{n}(z)=\phi_{k}(w)$. This can be taken to define an equivalence relation on $\mathbb{D}$, and then the grand orbits are the equivalent classes. Write $\mathcal{G}(\phi)$ for the set of all grand orbits for the self-map $\phi$. We do not worry here about what extra structures can be put on $\mathcal{G}(\phi)$. If $\sigma$ is a semiconjugation solving (1.3), then it sends grand orbits for $\phi$ into grand orbits for $\tau$, i.e., $\sigma$ induces a map $\Sigma$ from $\mathcal{G}(\phi)$ to $\mathcal{G}(\tau)$.

Remark 2.4. If $p \in \mathbb{D}$ is a fixed point of $\phi$, then $\sigma(p)$ is a fixed point of $\tau$.

Definition 2.5. We say that a solution $\sigma$ of (1.3) is canonical if the induced map $\Sigma$ from $\mathcal{G}(\phi)$ to $\mathcal{G}(\tau)$ is a bijection.

Proposition 2.6. The semiconjugation g obtained in Theorem A is canonical.

Proof. Write $\langle z\rangle$ for the grand orbit generated by $z$ and $\Sigma_{g}$ for the map induced by $g$ between the spaces of grand orbits.

In the hyperbolic case, recall that by Remark 2.3 every forward orbit $z_{n}$ for $\phi$ tends to infinity non-tangentially. Thus, given $\Omega$ as in Lemma 2.2 (a), every orbit is eventually in $\Omega$, so the grand orbits for $\phi$ are in one-to-one correspondence with the forward orbits in $\Omega$. Since $g$ is one-to-one on $\Omega$, we have that $\Sigma_{g}$ is one-to-one. Also, since $g(\Omega)$ has an inner-tangent at infinity, we also have that $\Sigma_{g}$ is onto the space of grand orbits for $\alpha$.

In the parabolic non-zero-step case, suppose that $\langle z\rangle \neq\langle w\rangle$. Then, referring to Lemma $2.2(\mathrm{~b}), z_{n}, w_{n} \in \Omega\left(\rho, m_{\rho}\right)$ for some $\rho$ and for $n$ sufficiently large. So $\Sigma_{g}(\langle z\rangle) \neq \Sigma_{g}(\langle w\rangle)$. On the other hand if an orbit of $\alpha$ is given, $\{\zeta+n b\}$, then $\zeta+n b \in g(U)$ for $n$ large. So $\Sigma_{g}$ is onto.

\section{MAin RESUlts}

Here we state our results regarding the classical semiconjugations in the hyperbolic and parabolic non-zero-step cases, we abbreviate these two cases by saying "non-zero-step type", see Remark 1.1. The other parabolic case will be discussed later in the paper.

First we need a result similar to Lemma 2.61 of [CM03]. 
Proposition 3.1. Suppose $\phi$ is a self-map of $\mathbb{H}$ of non-zero-step type, as in (1.1) with $A \geq 1$. Let $(g, \alpha)$ be the semiconjugation obtained in Theorem A. Given a solution $(\sigma, \tau) \in \mathcal{F}(\phi)$, there is an analytic self-map $F$ of $\mathbb{D}$ such that

$$
\sigma=F \circ g
$$

and

$$
F \circ \alpha=\tau \circ F \text {. }
$$

Moreover, $\sigma$ is canonical if and only if $F$ is.

Proposition 3.1 says that in order to solve the Forward Equation (1.3), it is enough to consider tha pair $(g, \alpha)$ of Theorem A, and then solve (1.3) with the self-map $\phi$ replaced by the automorphism $\alpha$. In particular, $F$ is a self-map of $\mathbb{D}$ which intertwines two automorphisms $\alpha$ and $\tau$. This fact is helpful in determining the canonical solutions to (1.3), because it is easier to determine when $F$ is canonical (this will be done below in Section 5). As a result we will obtain the following characterizations of canonical solutions and thus answer Question 1.4 .

Theorem 3.2. Assume $\phi$ is a self-map of $\mathbb{H}$ of non-zero-step type. Let $(g, \alpha), A, b$ be as in Theorem $A$, and let $\beta$ be as in Remark 1.6. Given a solution $(\sigma, \tau) \in \mathcal{F}(\phi)$ where $\tau$ is not elliptic, let $\tilde{\beta}$ be the automorphism of $\mathbb{H}$ that conjugates $\tau$ to its standard form. Then the following are equivalent:

(a) When $A>1, \tilde{\beta}^{-1} \circ \tau \circ \tilde{\beta}(z)=A z$, and there is $c>0$ so that $\sigma=\tilde{\beta} \circ\left(c \beta^{-1}\right) \circ g$.

When $A=1, \tilde{\beta}^{-1} \circ \tau \circ \tilde{\beta}(z)=z+1$, and there is $d \in \mathbb{R}$ such that $\sigma=\tilde{\beta}\left(\beta^{-1} \circ g+d\right)$.

(b) $\sigma$ is canonical (see Definition 2.5).

(c) $\tilde{\beta}^{-1} \circ \sigma$ has the same univalence and covering properties of $g$ in Lemma 2.2.

(d) There exists a pair of points $z, w \in \mathbb{H}$ for which equality holds in (2.1), not identically zero.

Corollary 3.3. Suppose $\phi$ is a self-map of $\mathbb{H}$ of non-zero-step type, written as in (1.1) with $A \geq 1$. Consider the semiconjugations $(g, \alpha(z)=A z+b)$ and $(\tilde{g}, \tilde{\alpha}(z)=A z+\tilde{b})$, obtained in Theorem $A$ by renormalizing $\phi_{n}$ using $\left\{\phi_{n}\left(z_{0}\right)\right\}$ and $\left\{\phi_{n}\left(\tilde{z}_{0}\right)\right\}$ respectively. Then, when $A>1$,

$$
\frac{\tilde{g}+\frac{\tilde{b}}{A-1}}{i+\frac{\tilde{b}}{A-1}}=\frac{g+\frac{b}{A-1}}{g\left(\tilde{z}_{0}\right)+\frac{b}{A-1}}
$$

while, when $A=1$,

$$
\frac{\tilde{g}-i}{|\tilde{b}|}=\frac{g-g\left(\tilde{z}_{0}\right)}{|b|}
$$




\section{THE FORWARD EQUATION}

We give the proof of Proposition 3.1 here and we postpone the proofs of Theorem 3.2 and Corollary 3.3 to a later section.

Proof of Proposition 3.1. Assume first that $\phi$ is of hyperbolic type. Let $\Omega$ be the simply connected domain in $\mathbb{H}$ with inner-tangent at infinity, such that $g$ is one-to-one on $\Omega$, which is given by Lemma 2.2 (a). Recall that $g(\Omega)$ also has an inner-tangent at infinity, and that $\phi \circ(g \mid \Omega)^{-1}(w)=(g \mid \Omega)^{-1}(\alpha(w))$ whenever $w, \alpha(w) \in g(\Omega)$.

Given $(\sigma, \tau) \in \mathcal{F}(\phi)$, define $F(w)=\sigma \circ(g \mid \Omega)^{-1}$ on $g(\Omega)$. Given $w \in \mathbb{H} \backslash \Omega$, let $n_{0}=n_{0}(w)$ be the smallest integer such that the iterates $\alpha_{n}(w)$ are in $\Omega$ for $n \geq n_{0}$. Then,

$$
\begin{aligned}
\tau_{n_{0}+k}^{-1} \circ F \circ \alpha_{n_{0}+k}(w) & =\tau_{n_{0}+k}^{-1} \circ \sigma \circ(g \mid \Omega)^{-1} \circ \alpha_{k} \circ \alpha_{n_{0}}(w) \\
& =\tau_{n_{0}}^{-1} \circ \tau_{k}^{-1} \circ \sigma \circ \phi_{k} \circ(g \mid \Omega)^{-1} \circ \alpha_{n_{0}}(w) \\
& =\tau_{n_{0}}^{-1} \circ \sigma \circ(g \mid \Omega)^{-1} \circ \alpha_{n_{0}}^{-1}(w) \\
& =\tau_{n_{0}}^{-1} \circ F \circ \alpha_{n_{0}}^{-1}(w)
\end{aligned}
$$

Therefore, $F$ is well-defined on all of $\mathbb{H}$ via the formula $F(w)=\tau_{n_{0}}^{-1} \circ \sigma \circ(g \mid \Omega)^{-1}\left(\alpha_{n_{0}}(w)\right)$, with the understanding that $n_{0}=0$ if $w \in \Omega$. Moreover, $F \circ \alpha=\tau \circ F$, so $F \in \mathcal{A}(\alpha, \tau)$.

Now assume that $\phi$ is of parabolic non-zero-step type. Let $(\sigma, \tau) \in \mathcal{F}(\phi)$, and let $w \in \mathbb{H}$. Pick $\rho=2 \rho_{\mathbb{H}}(w, i)$. By Lemma $2.2(\mathrm{~b})$, for $n \geq n(w), \alpha_{n}(w) \in g(U)$, and $(g \mid U)^{-1}\left(\alpha_{n+k}(w)\right)=\phi_{k}\left((g \mid U)^{-1}\left(\alpha_{n}(w)\right)\right)$, for $k=1,2,3, \ldots$ Thus, we define

$$
F(w)=\tau_{n}^{-1} \circ \sigma \circ(g \mid U)^{-1} \circ \alpha_{n}(w)
$$

for some $n \geq n(w)$, and this definition does not depend on which $n \geq n(w)$ is chosen. Then $F$ is an analytic self-map of $\mathbb{H}$, and $F \circ \alpha=\tau \circ F$. Moreover $F \circ g(z)=\sigma(z)$.

For the "moreover part", it is clear that $\sigma$ is canonical if and only if both $F$ and $g$ are, so the claim follows from Proposition 2.6.

The next section lists the possible intertwining maps between automorphisms of the disk, and identify the ones that are canonical.

\section{MAPs that intertwine AUTOMORPhisms OF THE DISK}

In this section we study the forward equation (1.3) when $\phi:=\gamma \in \operatorname{Aut}(\mathbb{D})$. Actually in this situation it does not make sense to distinguish between forward and backward.

Since there are three cases for $\gamma$ and three for $\tau$ this yields nine cases. However, by Remark 2.4, only seven may have non-trivial solutions. We are also using (1.6) and (1.7). 


\subsection{The cases.}

- $\mathcal{A}_{h \rightarrow h}(S, T)=\{\sigma: \mathbb{H} \rightarrow \mathbb{H} ; T, S>1, \mid \sigma(S z)=T \sigma(z)\}$.

- $\mathcal{A}_{h \rightarrow p}(S, \pm)=\{\sigma: \mathbb{H} \rightarrow \mathbb{H} ; S>1 \mid \sigma(S z)=\sigma \pm 1\}$.

- $\mathcal{A}_{h \rightarrow e}(S, \theta)=\left\{\sigma: \mathbb{H} \rightarrow \mathbb{D} ; S>1 ; \theta \in[0,2 \pi) \mid \sigma(S z)=e^{i \theta} \sigma(z)\right\}$.

- $\mathcal{A}_{p \rightarrow p}( \pm, \pm)=\{\sigma: \mathbb{H} \rightarrow \mathbb{H} \mid \sigma(z \pm 1)=\sigma(z) \pm 1\}$.

- $\mathcal{A}_{p \rightarrow h}( \pm, T)=\{\sigma: \mathbb{H} \rightarrow \mathbb{H} ; T>1 \mid \sigma(z \pm 1)=T \sigma(z)\}$.

- $\mathcal{A}_{p \rightarrow e}( \pm, \theta)=\left\{\sigma: \mathbb{H} \rightarrow \mathbb{D} ; \theta \in[0,2 \pi) \mid \sigma(z \pm 1)=e^{i \theta} \sigma\right\}$.

- $\mathcal{A}_{e \rightarrow e}(\varphi, \theta)=\left\{\sigma: \mathbb{D} \rightarrow \mathbb{D} ; \theta, \varphi \in[0,2 \pi) \mid \sigma\left(e^{i \varphi} z\right)=e^{i \theta} \sigma(z)\right\}$.

For simplicity, we again restrict our attention to $\gamma$ hyperbolic or parabolic to start with. The case $\mathcal{A}_{e \rightarrow e}$, when $\gamma$ is elliptic, is actually more complicated and will be postponed to a later section.

Proposition 5.1. We have

(1) $\mathcal{A}_{p \rightarrow p}( \pm, \pm)=\mathcal{A}_{p \rightarrow p}(+,+) \cup \mathcal{A}_{p \rightarrow p}(-,-)$. Moreover, $\mathcal{A}_{p \rightarrow p}(+,+)=\mathcal{A}_{p \rightarrow p}(-,-)$.

(2) $\mathcal{A}_{p \rightarrow h}( \pm, T)$ is empty.

(3) If $1<S<T$, then $\mathcal{A}_{h \rightarrow h}(S, T)$ is empty; if $1<T<S$, then every $\sigma \in \mathcal{A}_{h \rightarrow h}(S, T)$ satisfies n.t. $-\lim _{z \rightarrow \infty} \sigma(z) / z=0$; while if $T=S$, then $\mathcal{A}_{h \rightarrow h}(S, S)=\{c z \mid c>0\}$.

In all three cases in this proposition, $\sigma$ is a self-map of $\mathbb{H}$, so we can write

$$
\sigma(z)=c z+p(z)
$$

with $0 \leq c<\infty, \operatorname{Im} p(z)>0$ and n.t. $-\lim _{z \rightarrow \infty} p(z) / z=0$. We first observe that $\sigma(z) / z$ has the Lindelöf property, i.e., suppose $\gamma:[0,+\infty) \rightarrow \mathbb{H}$ is a path such that $\lim _{t \rightarrow+\infty} \gamma(t)=\infty$, and suppose

$$
\lim _{t \rightarrow+\infty} \frac{\sigma(\gamma(t))}{\gamma(t)}=a
$$

for some $a \in \mathbb{C} \cup\{\infty\}$. Then,

$$
a=\text { n. t. }-\lim _{z \rightarrow \infty} \frac{\sigma(z)}{z}=c .
$$

In fact, since $p(z) / z$ misses the negative real axis, composition with a conformal map to the disk will turn it into a bounded function. So (5.1) follows from the usual Lindelöf property of bounded analytic functions in $\mathbb{H}$, see [Ahl73] p. 40,

Proof of Proposition 5.1. In (1), suppose that $\sigma$ is a self-map of $\mathbb{H}$ such that $\sigma(z+1)=$ $\sigma(z)-1$ (the other case is similar). Let $\gamma_{n}(t)=i+n t, t \in[0,1], n=0,1,2,3 \ldots$, and $\Gamma=\cup \gamma_{n}$. Then, since $\sigma(z+n)=\sigma(z)-n$, the function $\sigma(z) / z$ has limit -1 along $\Gamma$. Therefore, by the Lindelöf's property, n.t. $-\lim _{z \rightarrow \infty} \sigma(z) / z=-1=c$, but $c \geq 0$.

The moreover part is trivial. 
In (2), suppose that $\sigma(z+1)=T \sigma(z)$ with $T>1$. Let $\Gamma$ be the path defined above. Then, since $\sigma(z+n)=T^{n} \sigma(z)$, the function $\sigma(z) / z$ tends to infinity along $\Gamma$. So, by the Lindelöf's property, n.t. $-\lim _{z \rightarrow \infty} \sigma(z) / z=\infty=c$, but $c<\infty$.

In (3), since $\sigma\left(S^{n} z\right)=T^{n} \sigma(z)$, we have

$$
\frac{\sigma\left(S^{n} z\right)}{S^{n} z}=\left(\frac{T}{S}\right)^{n} \frac{\sigma(z)}{z}=c+\frac{p\left(S^{n} z\right)}{S^{n} z} \rightarrow c .
$$

Thus $T \leq S$, and if $T<S$, then $c=0$. On the other hand, if $T=S$, then $\sigma(z) / z=c$, which implies that $c>0$ and that $\sigma(z)=c z$.

Remark 5.2. If $\sigma \in \mathcal{A}_{h \rightarrow h}(S, T)$, then $\log \sigma / \log T \in \mathcal{A}_{h \rightarrow p}(S,+)$ (here $\left.0<\operatorname{Im} \log z<\pi\right)$.

Also, if $\sigma \in \mathcal{A}_{h \rightarrow p}(S, \pm)$, then $e^{i \theta \sigma} \in \mathcal{A}_{h \rightarrow e}(S, \pm \theta)$. Conversely, if $\sigma \in \mathcal{A}_{h \rightarrow e}(S, \theta)$, and $\sigma \neq 0$, then $(\log \sigma) /(i \theta) \in \mathcal{A}_{h \rightarrow p}(S,+)$, for any choice of log. Examples can be found in $\mathcal{A}_{h \rightarrow e}(S, \theta)$ that do have zeros.

Finally, if $\sigma \in \mathcal{A}_{p \rightarrow p}( \pm, \pm)$, then $e^{i \theta \sigma} \in \mathcal{A}_{p \rightarrow e}( \pm, \pm \theta)$. Conversely, if $\sigma \in \mathcal{A}_{p \rightarrow e}(+, \theta)$, and $\sigma \neq 0$, then $(\log \sigma) /(i \theta) \in \mathcal{A}_{p \rightarrow p}(+,+)$, for any choice of $\log$. Examples can be found in $\mathcal{A}_{p \rightarrow e}(+, \theta)$ that do have zeros.

5.2. Canonical solutions. We next describe all the possible canonical solutions in the set $\mathcal{A}$ which comprises all the cases in Section 5.1 above, except $\mathcal{A}_{e \rightarrow e}$.

Theorem 5.3. The only canonical solutions arising in $\mathcal{A}$ are:

(1) $\{c z \mid c>0\}=\mathcal{A}_{h \rightarrow h}(S, S)$;

(2) $\{z+d \mid d \in \mathbb{R}\} \subset \mathcal{A}_{p \rightarrow p}( \pm, \pm)$.

Proof. We first list the possible grand orbit spaces that arise depending on the nature of $\gamma$ :

- $\mathcal{G}_{S}=\mathbb{H} /\langle z \mapsto S z\rangle(S>1)$;

- $\mathcal{G}_{+}=\mathbb{H} /\langle z \mapsto z+1\rangle$;

- $\mathcal{G}_{\theta}=\mathbb{D} /\left\langle z \mapsto e^{i \theta} z\right\rangle(\theta \in(0,2 \pi))$.

Notice that $\mathcal{G}_{S}$ is a Riemann surface conformally equivalent to an annulus, and $\mathcal{G}_{+}$is a Riemann surface conformally equivalent to a punctured disk. Moreover $\mathcal{G}_{S}$ is not conformally equivalent to $\mathcal{G}_{+}$, and if $1<T<S$, then $\mathcal{G}_{S}$ is not conformally equivalent to $\mathcal{G}_{T}$.

A solution $\sigma$ in $\mathcal{A}_{h \rightarrow h}(S, T)$ induces an analytic map $\Sigma$ from $\mathcal{G}_{S}$ to $\mathcal{G}_{T}$. Imposing that $\sigma$ is canonical, means that $\Sigma$ is a bijection, and since $\Sigma$ is analytic, this automatically implies that $\Sigma$ is biholomorphic. Thus, $\mathcal{G}_{S}$ is conformally equivalent to $\mathcal{G}_{T}$, and therefore $S=T$.

Likewise, a solution $\sigma$ in $\mathcal{A}_{h \rightarrow p}(S,+)$ factors down to an analytic map $\Sigma$ between $\mathcal{G}_{S}$ and $\mathcal{G}_{+}$, and since these two Riemann surfaces are not conformally equivalent, none of these solutions $\sigma$ are canonical. 
Finally, suppose $\sigma \in \mathcal{A}_{p \rightarrow p}(+,+)$ induces an automorphism on $\mathcal{G}_{+}$. Then $\sigma \in \operatorname{Aut}(\mathbb{H})$ and actually $\sigma \in \operatorname{Aut}_{\infty}(\mathbb{H})$. So $\sigma(z)=c z+d$, with $c>0$ and $d \in \mathbb{R}$. However, since $\sigma(z+1)=\sigma(z)+1$, we must have $c=1$. Hence, $\sigma(z)=z+d$ and these are all the possible canonical solutions that can be found in $\mathcal{A}_{p \rightarrow p}(+,+)$.

The case of $\mathcal{G}_{\theta}$ is not so straightforward. Suppose $\sigma$ is in $\mathcal{A}_{h \rightarrow e}(S, \theta)$, or in $\mathcal{A}_{p \rightarrow e}( \pm, \theta)$, and assume that $\sigma$ is canonical. When $\theta /(2 \pi) \in \mathbb{Q}$, we still obtain a Riemann surface $\mathcal{G}_{\theta}^{\star}=(\mathbb{D} \backslash\{0\}) /\left\langle z \mapsto e^{i \theta} z\right\rangle$ which is conformally equivalent to a punctured disk. Since $\sigma$ is canonical it must be non-constant. Letting $Z=\sigma^{-1}(0) \subset \mathbb{H}$, we see that $\sigma$ factors down to a conformal equivalence between the punctured disk $\mathcal{G}_{\theta}^{\star}$ and either $\mathcal{G}_{S} \backslash P$ or $\mathcal{G}_{+} \backslash Q$, where $P$ and $Q$ are non-empty discrete sets of punctures, corresponding to $Z$. Since no such equivalence exists, $\sigma$ cannot be canonical.

Now assume that $\theta /(2 \pi) \notin \mathbb{Q}$. Then $\sigma(z)=\sigma(w)$ implies that $z$ and $w$ generate the same grand orbit. Therefore, there is $k \in \mathbb{Z}$ such that $w=S^{k} z$, or $w=z+k$. Thus, $\sigma(z)=e^{i k \theta} \sigma(z)$, so $\sigma(z)=\sigma(w)=0$. Then $\sigma$ vanishes on an infinite discrete set $Z$, yet is one-to-one on $\mathbb{H} \backslash Z$, and this contradicts the argument principle.

\section{Canonical Semiconjugations For SElF-MaPs With NON-ZERO STEP}

In this section we prove Theorem 3.2 and Corollary 3.3.

Proof of Theorem 3.2. The implication $(a) \Rightarrow(b)$ follows from Proposition 2.6, since $\sigma$ is equal to $g$ post-composed by an automorphism of $\mathbb{H}$.

Conversely, by Proposition 3.1 any solution $\sigma$ can be written as $\sigma=F \circ g$, with $F \circ \alpha=\tau \circ F$, and $\sigma$ is canonical if and only if $F$ is. By (1.6) and (1.7)

$$
(F, \tau) \in \mathcal{F}(\alpha) \Longleftrightarrow\left(\tilde{\beta}^{-1} \circ F \circ \beta, \tilde{\beta}^{-1} \circ \tau \circ \tilde{\beta}\right) \in \mathcal{F}\left(\beta^{-1} \circ \alpha \circ \beta\right)
$$

Choose $\tilde{\beta}$ so that $\tilde{\beta}^{-1} \circ \tau \circ \tilde{\beta}$ is in standard form as in Section 1.1. Notice that $\tilde{\beta}^{-1} \circ F \circ \beta$ is canonical, and when $A>1$ it intertwines $z \mapsto A z$ with $\tilde{\beta}^{-1} \circ \tau \circ \tilde{\beta}$, which is some other automorphism of $\mathbb{H}$ in standard form. Therefore Theorem 5.3 implies that $\tilde{\beta}^{-1} \circ F \circ \beta=c z$ for some $c>0$, and also that $\tilde{\beta}^{-1} \circ \tau \circ \tilde{\beta}(z)=A z$. So, $F(z)=\tilde{\beta}\left(c \beta^{-1}(z)\right)$. In particular, $\tilde{\beta}^{-1} \circ \sigma=c \beta^{-1} \circ \mathrm{g}$. On the other hand, when $A=1$, we assume without loss of generality that $b>0$. Then $\tilde{\beta}^{-1} \circ F \circ \beta$ intertwines $z \mapsto z+1$ with $\tilde{\beta}^{-1} \circ \tau \circ \tilde{\beta}$. So by Theorem 5.3, $\tilde{\beta}^{-1} \circ F \circ \beta=z+d$ for some $d \in \mathbb{R}$, and also that $\tilde{\beta}^{-1} \circ \tau \circ \tilde{\beta}(z)=z+1$. So, $F(z)=\tilde{\beta}\left(\beta^{-1}(z)+d\right)$. In particular, $\tilde{\beta}^{-1} \circ \sigma=\beta^{-1} \circ g+d$. Hence, we have shown $(b) \Rightarrow(a)$.

Note that $(a) \Rightarrow(c)$ is clear from Lemma 2.2. Conversely, by Proposition 3.1 we can write $\sigma=F \circ g$, with $F \circ \alpha=\tau \circ F$. We claim that $F$ must be an automorphism of $\mathbb{H}$. This implies that $\sigma$ is canonical, so $(c) \Rightarrow(a)$. To see that $F$ is one-to-one, suppose $F(z)=F(w)$. Then

$$
F\left(\alpha_{n}(z)\right)=\tau_{n} \circ F(z)=\tau_{n} \circ F(w)=F\left(\alpha_{n}(w)\right)
$$


Find $\rho>0$ large enough so that $z, w \in \Delta(i, \rho)$. By the proof of the 'furthermore' part in Lemma 2.2, we can choose $N$ large enough so that $\alpha_{n}(z), \alpha_{n}(w) \in g\left(\Delta\left(z_{n}, \rho\right)\right)$, and so that $g$ is univalent on $\Delta\left(z_{n}, \rho\right)$, for all $n \geq N$. By hypothesis, we can find $n$ large enough so that $\sigma=F \circ g$ is univalent on $\Delta\left(z_{n}, \rho\right)$. Thus $\alpha_{n}(z)=\alpha_{n}(w)$, and $z=w$.

To see that $F$ is onto, recall that, by hypothesis, $\tilde{\beta}^{-1} \circ \sigma$ has certain univalence and covering properties at $\infty$. Thus, $\tilde{\beta}^{-1} \circ F$ must have certain corresponding properties. Hence we can define

$$
G(z):=\alpha_{n}^{-1} \tilde{F}^{-1}\left(\tilde{\tau}_{n}^{-1}(z)\right)
$$

to be a global analytic inverse of $\tilde{F}$ in $\mathbb{H}$, where $\tilde{\tau}=\tilde{\beta}^{-1} \circ \tau \circ \tilde{\beta}$, and $n$ is chosen large enough depending on $z$.

Finally, $(a) \Rightarrow(d)$ is clear from (2.1). Conversely, write $\sigma=F \circ g$. Then,

$$
\rho_{\mathbb{H}}(F(g(z)), F(g(w)))=\rho_{\mathbb{H}}(g(z), g(w)) \neq 0 .
$$

So, by Schwarz's Lemma, $F$ is an automorphism of $\mathbb{H}$. Hence, $\sigma$ is canonical, and $(d) \Rightarrow$ $(a)$.

Proof of Corollary 3.3. Let $\sigma=\tilde{g}$ and $\tau=\tilde{\alpha}$, then apply Theorem 3.2 and the fact that $\tilde{g}$ is canonical. Then plug in $z=\tilde{z}_{0}$.

\section{The PARABOliC ZERO-STEP CASE}

Proposition 2.1 shows that when $\phi$ is parabolic zero-step, then $\mathcal{F}(\phi)$ is empty. So instead of looking for pairs $(\sigma, \tau)$ where $\sigma$ is a self-map of $\mathbb{H}$ and $\tau$ is an automorphism of $\mathbb{H}$, one is led to try to find pairs where $\sigma$ is analytic and $\tau$ is linear, namely solutions to the Planar Equation.

Definition 7.1. We write $\mathcal{P}(\phi)$ for the family of all non-trivial solution pairs $(\sigma, \tau)$ to $(1.5)$, i.e., we ask that $\sigma$ be non-constant.

Note that (1.6) and (1.7) still hold with $\mathcal{F}$ replaced by $\mathcal{P}$ and $\alpha, \beta \in \operatorname{Aut}(\mathbb{C})$. So $\tau$ can always be put in standard form: namely, either $\tau(z)=a z$ for some $a \in \mathbb{C} \backslash\{0\}$, or $\tau(z)=z+1$. Again we will write

- $\mathcal{P}_{e}(\phi, a)=\{\sigma: \mathbb{H} \rightarrow \mathbb{C} ; a \in \mathbb{C} \backslash\{0\} \mid \sigma \circ \phi=a \sigma\}$.

- $\mathcal{P}_{p}(\phi,+)=\{\sigma: \mathbb{H} \rightarrow \mathbb{C} \mid \sigma \circ \phi=\sigma+1\}$.

When $\phi$ is parabolic zero-step, existence of solutions in $\mathcal{P}_{p}(\phi,+)$ is found in [BP79]. In [Cow81] this dichotomy between disk model and plane model is explained in the framework of the "type problem" for simply-connected non-compact Riemann surfaces and the uniformization theorem. 
Fix a self-map of $\mathbb{H}$ of parabolic zero-step type, which can therefore be written as

$$
\phi(z)=z+p(z)
$$

$\operatorname{Im} p(z)>0$ and n.t. $\lim _{z \rightarrow \infty} p(z) / z=0$ (see the classification in Section 1.1). Write $z_{n}=$ $\phi_{n}\left(z_{0}\right)=x_{n}+i y_{n}$, and let

$$
\mu_{n}(z):=\frac{z-z_{n}}{z_{n+1}-z_{n}} \in \operatorname{Aut}(\mathbb{C})
$$

be the automorphism of $\mathbb{C}$ which sends $z_{n}$ to 0 and $z_{n+1}$ to 1 . Then consider the normalized iterates

$$
h_{n}=\mu_{n} \circ \phi_{n} .
$$

Theorem B (Theorem 1 of [BP79]). The limit $h=\lim _{n \rightarrow \infty} h_{n}$ exists locally uniformly in $\mathbb{H}$, satisfies $h\left(z_{0}\right)=0$, and solves

$$
h \circ \phi=h+1 \text {. }
$$

Remark 7.2. The zero-step condition implies that

$$
\frac{y_{n+1}}{y_{n}} \longrightarrow 1 \quad \text { and } \quad \frac{x_{n+1}-x_{n}}{y_{n}} \longrightarrow 0,
$$

and again $y_{n+1}>y_{n}$ so $y_{n} \rightarrow L_{\infty}$. Using the zero-step hypothesis one sees that $L_{\infty}=+\infty$.

We now describe the univalence and covering properties of the semiconjugation $h$ in the spirit of Section 2.2.

Lemma 7.3. The sequence $\psi_{n}=h \circ \mu_{n}^{-1}-n$ converges uniformly on compact subsets of $\mathbb{C}$ to the identity map on $\mathbb{C}$. Moreover, sequences $R_{k}, M_{k} \uparrow+\infty$ can be chosen so that $h$ is univalent on the set

$$
U=\bigcup_{k=1}^{\infty} \bigcup_{n=M_{k}}^{\infty}\left(z_{n}+\left(z_{n+1}-z_{n}\right) R_{k} \mathbb{D}\right)
$$

Furthermore, the region $V=h(U)$ has a lateral-tangent at $+\infty$ with respect to $\mathbb{C}$, i.e., given $R_{n} \uparrow+\infty$ there is $t_{n} \uparrow+\infty$ such that $\left\{\operatorname{Re} z>t_{n},|\operatorname{Im} z|<R_{n}\right\} \subset V$.

Proof. First recall part (c) of the proof of the main theorem in [BP79], where it shown that there is $m$ so that $h\left(z_{n}+y_{n} s\right)$ is univalent in $|s|<1 / 5$ for all $n \geq m$. Since $\mu_{n}^{-1}(\zeta)=$ $z_{n}+\zeta\left(z_{n+1}-z_{n}\right)$, and since by Remark 7.2

$$
\frac{z_{n+1}-z_{n}}{y_{n}}=\frac{x_{n+1}-x_{n}}{y_{n}}+i\left(\frac{y_{n+1}}{y_{n}}-1\right) \rightarrow 0
$$

we see that given $R>1$ there is $N=N(R) \in \mathbb{N}$ such that

$$
\psi_{n}(\zeta)=h \circ \mu_{n}^{-1}(\zeta)-n=h\left(z_{n}+\zeta\left(z_{n+1}-z_{n}\right)\right)-n
$$

is well defined and univalent for all $|\zeta|<R$ and all $n \geq N$. 
Moreover, $\psi_{n}(0)=h\left(z_{n}\right)-n=0$ and $\psi_{n}(1)=h\left(z_{n+1}\right)-n=1$. So the sequence $\left\{\psi_{n}\right\}$ is normal on $\{|\zeta|<R\} \backslash\{0,1\}$, and hence, by a standard argument, on $\{|\zeta|<R\}$ as well. Now,

$$
\psi_{n}\left(h_{n}\right)=h \circ \mu_{n}^{-1} \circ \mu \circ \phi_{n}-n=h
$$

So any normal sublimit $\psi$ of the $\psi_{n}$ is the identity map on $h(\mathbb{H})$. But since $h$ is an open map, this implies that $\psi_{n}$ converges to the identity on $\mathbb{C}$.

To prove the univalence statement we proceed as in the proof of Theorem 3 in [Pom79]. First note that, since $\psi_{n}$ tends to the identity map uniformly on compact sets, for every $R>1$ there is an integer $k_{R}$ such that

$$
\left(\psi_{n}(R \mathbb{D})\right) \cap\left(\psi_{n+k_{R}}(R \mathbb{D})+k_{R}\right)=\emptyset
$$

for $n=1,2,3, \ldots$

Now suppose that $h\left(s_{1}\right)=h\left(s_{2}\right)$ at points $s_{1}=\mu_{n}^{-1}\left(\zeta_{1}\right)$ and $s_{2}=\mu_{n+j}^{-1}\left(\zeta_{2}\right)$ with $j \geq 0$ and $\left|\zeta_{l}\right|<R$ for $l=1,2$. Then

$$
\begin{aligned}
\psi_{n}\left(\zeta_{1}\right) & =h \circ \mu_{n}^{-1}\left(\zeta_{1}\right)-n=h\left(s_{1}\right)-n \\
& =h\left(s_{2}\right)-n=h \circ \mu_{n+j}^{-1}\left(\zeta_{2}\right)-(n+j)+j=\psi_{n+j}\left(\zeta_{2}\right)+j
\end{aligned}
$$

So by (7.3) we must have $j \leq k_{R}$.

On the other hand,

$$
s_{2}=z_{n+j}+\left(z_{n+j+1}-z_{n+j}\right) \zeta_{2}=z_{n}+\left(z_{n+1}-z_{n}\right) t_{n}
$$

where

$$
t_{n}=\frac{\left(z_{n+j}-z_{n}\right)+\left(z_{n+j+1}-z_{n+j}\right) \zeta_{2}}{z_{n+1}-z_{n}} \rightarrow j-\zeta_{2}
$$

as $n \rightarrow \infty$. So $s_{2}, s_{1} \in \mu_{n}^{-1}\left(R^{\prime} \mathbb{D}\right)$ with $R^{\prime}=R+k_{R}+1$. However, since $\psi_{n}$ converges to the identity map on compact sets, there is $m_{R}$ such that $h$ is one-to-one on $\mu_{n}^{-1}\left(R^{\prime} \mathbb{D}\right)$ for all $n \geq m_{R}$. Therefore, we must have $n<m_{R}$. In other words, $h$ is one-to-one on $\cup_{n \geq m_{R}} \mu_{n}^{-1}(R \mathbb{D})$. Thus, given a ball $R \mathbb{D}$, there is $m_{R} \in \mathbb{N}$ so that $h$ is univalent on the set

$$
U\left(R, m_{R}\right)=\bigcup_{n=m_{R}}^{\infty}\left(z_{n}+\left(z_{n+1}-z_{n}\right) R \mathbb{D}\right)=\bigcup_{n=m_{R}}^{\infty} \mu_{n}^{-1}(R \mathbb{D})
$$

The 'moreover' part is proved exactly as in the proof of Lemma 2.2 .

For the 'furthermore' part, consider the half-strips $S_{t}=\{\operatorname{Re} z>t,|\operatorname{Im} z|<R / 2\}$. Then by Lemma 7.3, given $\epsilon<R / 2$ there is $N$ such that, for all $n \geq N$,

$$
\left|\psi_{n}(\zeta)-\zeta\right|=\left|h\left(z_{n}+\zeta\left(z_{n+1}-z_{n}\right)\right)-(\zeta+n)\right|<\epsilon
$$

uniformly for $|\zeta| \leq R$. In particular, when $n>\max \left\{N, m_{R}\right\}$, for every $|w|<R / 2, h \circ$ $\mu_{n}^{-1}(\{|\zeta|=R\})$ winds around $w+n$ exactly once, i.e., $w+n \in h(U)$. Therefore, $S_{t} \subset h(U)$ for $t$ large enough. 
Next we prove the equivalent of Proposition 2.6.

Proposition 7.4. The semiconjugation h obtained in Theorem B is canonical.

Proof. We need to show that the map $\Sigma_{h}$ which takes grand orbits of $\phi$ to the orbits of $z \mapsto z+1$ on $\mathbb{C}$ is one-to-one and onto. Again write $\langle z\rangle$ for the grand orbit of $\phi$ generated by $z$. Suppose that $\langle z\rangle \neq\langle w\rangle$. Write $z_{n}=\phi_{n}(z)$ and $w_{n}=\phi_{n}(w)$. Then $h\left(z_{n}\right)=h(z)+n$ and $h\left(w_{n}\right)=h(w)+n$. So, by Lemma 7.3, there is $R$ large enough so that for $n$ large enough $z_{n}, w_{n} \in U\left(R, m_{R}\right)$. Since $z_{n+k} \neq w_{n}$ for all such $n$ 's and $k \geq 0$, the univalence of $h$ implies that $\Sigma_{h}(\langle z\rangle) \neq \Sigma_{h}(\langle w\rangle)$. On the other hand given an orbit $\{a+n\}_{n=-\infty}^{+\infty}$ with $a \in \mathbb{C}$, there is $N$ such that $a+n \in V=h(U)$ for $n \geq N$. So $\Sigma_{h}$ is onto.

We now state a result equivalent to Proposition 3.1.

Proposition 7.5. Suppose $\phi$ is a self-map of $\mathbb{H}$ of parabolic zero-step type. Let $h$ be the semiconjugation obtained in Theorem B. Given a solution $(\sigma, \tau) \in \mathcal{P}(\phi)$, there is an entire function F such that

$$
\sigma=F \circ h
$$

and

$$
F(z+1)=\tau \circ F(z)
$$

Moreover, $\sigma$ is canonical if and only if $F$ is.

Proof. The proof is exactly the same as the one for Proposition 3.1.

Next, we state a result equivalent to Theorem 3.2 .

Theorem 7.6. Assume that $\phi$ is a self-map of $\mathbb{H}$ of parabolic zero-step type. Let $h$ be given as in Theorem B. Given a solution $(\sigma, \tau) \in \mathcal{P}(\phi)$, let $\tilde{\beta}$ be the automorphism of $\mathbb{C}$ which conjugates $\tau$ to its standard form (either $z+1$ or az). Then the following are equivalent.

(a) There is $b \in \mathbb{C}$ such that $\tau(z)=z+b, \tilde{\beta}(z)=b z$, and $\sigma=b(h+c)$ for some $c \in \mathbb{C}$.

(b) $\sigma$ is canonical.

(c) $\tilde{\beta}^{-1} \circ \sigma$ has the same univalence and covering properties as $h$ in Lemma 7.3 .

During writing of this paper, we were informed that Contreras, Diaz-Madrigal, and Pommerenke have also proved a statement similar to $(\mathrm{a}) \Leftrightarrow(\mathrm{c})$ above, but with a different univalence requirement, see $[\mathrm{CDMP}]$.

Corollary 7.7. Suppose $\phi$ is a self-map of $\mathbb{H}$ of parabolic zero-step type. Let $h$ and $\tilde{h}$ be semiconjugations obtained as in Theorem B, by renormalizing $\phi_{n}$ using $\left\{\phi_{n}\left(z_{0}\right)\right\}$ and $\left\{\phi_{n}\left(\tilde{z}_{0}\right)\right\}$ respectively. Then, $\tilde{h}(z)=h(z)-h\left(\tilde{z}_{0}\right)$. 
7.1. Entire functions that intertwine automorphisms of the plane. This section parallels Section 5. Three cases arise.

- $\mathcal{E}_{p \rightarrow p}=\{F$ entire $\mid F(z+1)=F(z)+1\}$;

- $\mathcal{E}_{p \rightarrow e}(a)=\{F$ entire $; a \in \mathbb{C} \backslash\{0\} \mid F(z+1)=a F(z)\}$;

- $\mathcal{E}_{e \rightarrow e}(a, b)=\{F$ entire $; a, b \in \mathbb{C} \backslash\{0\} \mid F(a z)=b F(z)\}$.

Note that there cannot be $F$ entire such that $F(a z)=F(z)+1$, because at $z=0$, we would have $F(0)=F(0)+1$, which is impossible. So $\mathcal{E}_{e \rightarrow p}$ is empty. Also, we will not be interested in $\mathcal{E}_{e \rightarrow e}(a, b)$ for the moment, because our immediate concern is proving Theorem 7.6.

Next we analyze the canonical maps.

Theorem 7.8. The only canonical maps in $\mathcal{E}_{p \rightarrow p}$ are $F(z)=z+c, c \in \mathbb{C}$. Moreover, $\mathcal{E}_{p \rightarrow e}(a)$ does not contain any canonical maps.

Proof. Suppose $F \in \mathcal{E}_{p \rightarrow p}$, then $F$ induces a map $\Sigma$ from $\mathbb{C} \backslash\langle z \mapsto z+1\rangle$ to itself, which is analytic. Imposing that $\Sigma$ be one-to-one and onto, means that $\Sigma$ is an automorphism of $\mathbb{C} \backslash\langle z \mapsto z+1\rangle$ and therefore $F$ must also be an automorphism of $\mathbb{C}$. Thus, $F(z)=d z+c$ and since it must also intertwine $z \mapsto z+1$ with itself, we have $d=1$.

For the 'Moreover' part, assume first that $|a| \neq 1$. Then, is a compact Riemann surface. Hence there are no biholomorphisms between $\mathbb{C} \backslash\langle z \mapsto z+1\rangle$ and $\mathbb{C} \backslash\langle z \mapsto a z\rangle$. If $|a|=1$, assume first that $a=e^{i \theta}$ with $\theta /(2 \pi) \in \mathbb{Q}$. Let $Z=F^{-1}(0) \in \mathbb{C}$. Then $F$ canonical would induce a biholomorphism between a punctured infinite cylinder and the punctured plane. Since there are no such maps, the conclusion holds. Now assume that $\theta /(2 \pi) \notin \mathbb{Q}$. Then $F(z)=F(w)$ and $F$ canonical imply that $z$ and $w$ generate the same grand orbit. So there is $n \in \mathbb{N}$, such that, say, $w=z+n$. Hence $F(z)=e^{i n \theta} F(z)$, so $F(z)=F(w)=0$. Then $F$ vanishes on an infinite discrete set $Z$, yet is one-to-one on $\mathbb{C} \backslash Z$, and this contradicts the argument principle.

\subsection{Canonical semiconjugations for self-maps of parabolic zero-step type.}

Proof of Theorem 7.6. The implication (a) $\Rightarrow(\mathrm{b})$ follows from Proposition 7.4. Conversely, by Proposition 7.5, any solution $\sigma$ can be written as $\sigma=F \circ h$, with $F(z+1)=\tau \circ F(z)$, and $\sigma$ is canonical if and only if $F$ is, if and only if $\tilde{\beta}^{-1} \circ F$ is. Moreover, $\tilde{\beta}^{-1} \circ F$ intertwines $z \mapsto z+1$ with either $z \mapsto z+1$ or $z \mapsto a z(a \neq 0)$. Hence, by Theorem $7.8, \tilde{\beta}^{-1} \circ F$ is canonical implies $\tilde{\beta}^{-1} \circ F(z)=z+c$, for some $c \in \mathbb{C}$, and $\tilde{\beta}^{-1} \circ \tau \circ \tilde{\beta}(z)=z+1$. So $\tau(z)=z+b, \tilde{\beta}(z)=b z$, and $F(z)=b z+b c$. Therefore, $(\mathrm{b}) \Rightarrow(\mathrm{a})$.

The implication (a) $\Rightarrow$ (c) follows from Lemma 7.3. Conversely, by Proposition 7.5, we can write $\sigma=F \circ h$, with $F(z+1)=\tau \circ F(z)$. Using the covering and univalence properties 
we then show that $F$ must be an automorphism of $\mathbb{C}$ in exactly the same way as in the proof of Theorem 3.2 (c) $\Rightarrow$ (a).

Proof of Corollary 7.7. Let $\sigma=\tilde{g}$, then apply Theorem 7.6 and the fact that $\tilde{g}$ is canonical. Then plug in $z=\tilde{z}_{0}$.

\section{REFERENCES}

[Ahl73] Lars V. Ahlfors. Conformal invariants: topics in geometric function theory. McGraw-Hill Book Co., New York, 1973. McGraw-Hill Series in Higher Mathematics.

[BP79] I. N. Baker and Ch. Pommerenke. On the iteration of analytic functions in a halfplane. II. $J$. London Math. Soc. (2), 20(2):255-258, 1979.

[BPC03] Filippo Bracci and Pietro Poggi-Corradini. On Valiron's theorem. In Future trends in geometric function theory, RNC Workshop Jyväskylä, volume 92, pages 39-55. University of Jyväskylä, Department of Mathematics and Statistics, 2003.

[BS97] Paul S. Bourdon and Joel H. Shapiro. Cyclic phenomena for composition operators. Mem. Amer. Math. Soc., 125(596):x+105, 1997.

[CDMP] M. Contreras, S. Diaz-Madrigal, and Ch. Pommerenke. Some remarks on Abel equation in the unit disk. Preprint.

[CM03] Carl C. Cowen and Barbara D. MacCluer. Schroeder's equation in several variables. Taiwanese J. Math., 7(1):129-154, 2003.

[Cow81] Carl C. Cowen. Iteration and the solution of functional equations for functions analytic in the unit disk. Trans. Amer. Math. Soc., 265(1):69-95, 1981.

[PC00] Pietro Poggi-Corradini. Canonical conjugations at fixed points other than the Denjoy-Wolff point. Ann. Acad. Sci. Fenn. Math., 25(2):487-499, 2000.

[PC03] Pietro Poggi-Corradini. Backward-iteration sequences with bounded hyperbolic steps for analytic self-maps of the disk. Rev. Mat. Iberoamericana, 19(3):943-970, 2003.

[Pom79] Ch. Pommerenke. On the iteration of analytic functions in a halfplane. J. London Math. Soc. (2), 19(3):439-447, 1979.

[Val31] Georges Valiron. Sur l'itération des fonctions holomorphes dans un demi-plan. Bulletin Sc. math., 55(2):105-128, 1931.

[Val54] Georges Valiron. Fonctions analytiques. Presses Universitaires de France, Paris, 1954.

Department of Mathematics, Cardwell Hall, Kansas State University, Manhattan, KS 66506

E-mail address: pietro@math.ksu.edu 